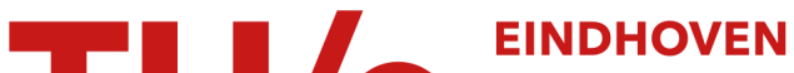 UNIVERSITY OF TECHNOLOGY
}

\section{Tail of the contact force distribution in static granular materials}

\section{Citation for published version (APA):}

Eerd, van, A. R. T., Ellenbroek, W. G., Hecke, van, M., Snoeijer, J. H., \& Vlugt, T. J. H. (2007). Tail of the contact force distribution in static granular materials. Physical Review E - Statistical, Nonlinear, and Soft Matter Physics, 75(6), 060302-1/4. [060302]. https://doi.org/10.1103/PhysRevE.75.060302

DOI:

10.1103/PhysRevE.75.060302

Document status and date:

Published: 01/01/2007

\section{Document Version:}

Publisher's PDF, also known as Version of Record (includes final page, issue and volume numbers)

\section{Please check the document version of this publication:}

- A submitted manuscript is the version of the article upon submission and before peer-review. There can be important differences between the submitted version and the official published version of record. People interested in the research are advised to contact the author for the final version of the publication, or visit the $\mathrm{DOI}$ to the publisher's website.

- The final author version and the galley proof are versions of the publication after peer review.

- The final published version features the final layout of the paper including the volume, issue and page numbers.

Link to publication

\section{General rights}

Copyright and moral rights for the publications made accessible in the public portal are retained by the authors and/or other copyright owners and it is a condition of accessing publications that users recognise and abide by the legal requirements associated with these rights.

- Users may download and print one copy of any publication from the public portal for the purpose of private study or research.

- You may not further distribute the material or use it for any profit-making activity or commercial gain

- You may freely distribute the URL identifying the publication in the public portal.

If the publication is distributed under the terms of Article 25fa of the Dutch Copyright Act, indicated by the "Taverne" license above, please follow below link for the End User Agreement:

www.tue.nl/taverne

Take down policy

If you believe that this document breaches copyright please contact us at:

openaccess@tue.nl

providing details and we will investigate your claim. 


\title{
Tail of the contact force distribution in static granular materials
}

\author{
Adrianne R. T. van Eerd, ${ }^{1}$ Wouter G. Ellenbroek, ${ }^{2}$ Martin van Hecke, ${ }^{3}$ Jacco H. Snoeijer, ${ }^{4}$ and Thijs J. H. Vlugt ${ }^{1}$ \\ ${ }^{1}$ Condensed Matter and Interfaces, Utrecht University, P.O. Box 80.000, 3508 TA Utrecht, The Netherlands \\ ${ }^{2}$ Instituut-Lorentz, Universiteit Leiden, P.O. Box 9506, 2300 RA Leiden, The Netherlands \\ ${ }^{3}$ Kamerlingh Onnes Lab, Leiden University, P.O. Box 9504, 2300 RA Leiden, The Netherlands \\ ${ }^{4}$ School of Mathematics, University of Bristol, University Walk, Bristol BS8 1TW, United Kingdom
}

(Received 20 February 2007; published 29 June 2007)

\begin{abstract}
We numerically study the distribution $P(f)$ of contact forces in frictionless bead packs, by averaging over the ensemble of all possible force network configurations. We resort to umbrella sampling to resolve the asymptotic decay of $P(f)$ for large $f$, and determine $P(f)$ down to values of order $10^{-45}$ for ordered and disordered systems in two (2D) and three dimensions (3D). Our findings unambiguously show that, in the ensemble approach, the force distributions decay much faster than exponentially: $P(f) \sim \exp \left(-c f^{\alpha}\right)$, with $\alpha \approx 2.0$ for $2 \mathrm{D}$ systems, and $\alpha \approx 1.7$ for $3 \mathrm{D}$ systems.
\end{abstract}

DOI: $10.1103 /$ PhysRevE.75.060302

The contact forces inside a static packing of grains are organized into highly heterogeneous force networks, and can be characterized by the probability density of contact forces $P(f)[1]$. Such force statistics were first studied in a series of experiments that measured forces through imprints on carbon paper at the boundaries of a granular assembly. Unexpectedly, the obtained $P(f)$ displayed an exponential rather than a Gaussian decay for large forces [2]. After these initial findings, other experimental techniques have revealed similarly exponentially decaying distributions of the boundary forces $[3,4]$.

As it is difficult to experimentally access contact forces inside the packing, numerous direct numerical simulations of $P(f)$ have been undertaken $[5,6]$. While many of these studies claim to find an exponential tail as well, the evidence is less convincing than for the carbon paper experiments: apart from [5], nearly all numerical force probabilities bend down on a logarithmic plot, suggesting a faster than exponential decay [6]. In addition, new experimental techniques using photoelastic particles [7] or emulsions $[8,9]$ have produced bulk measurements, and these also reveal a much faster than exponential decay for $P(f)$, consistent with a Gaussian tail.

Nevertheless, much theoretical effort has focused on explaining the exponential tail of $P(f)$, starting with the pioneering $q$ model [10]. Here, scalar forces are balanced on a regular grid, but it was later realized that, in this model, the tail of $P(f)$ depends on details of the stochastic rules for the force transmission, and need not be exponential [11]. Other explanations for the exponential tail hinge on "entropy maximization" [12], or closely related, on an analogy with the Boltzmann distribution $[13,14]$. The essence of the latter argument is that a uniform sampling of forces that (i) are all positive (corresponding to the repulsive nature of contact forces), and (ii) add up to a constant value (set by the requirement that the overall pressure is constant) strongly resembles the microcanonical ensemble, in which configurations are flatly sampled under the constraint of fixed total energy.

In this paper, we will probe the tail of $P(f)$ in the force network ensemble [15-20]. This ensemble is obtained by flatly sampling all force configurations for which forces are repulsive and add up to satisfy overall stresses, i.e., (i) and
PACS number(s): 45.70.Cc, 05.40.-a, 46.65.+g

(ii) as listed above, under the additional constraints of force balance on all grains. We numerically resolve the probability for large forces using the technique of umbrella sampling [21], which yields accurate statistics for $P(f)$ for relative probabilities down to $10^{-45}$ and $f$ up to $f=15$ (throughout, all forces are normalized such that $\langle f\rangle=1$ ). This high accuracy is crucial for excluding any crossover effects and allows us to unambiguously identify the behavior for $f \gg 1$. We study the force ensemble for frictionless systems in two and three dimensions, with both ordered and disordered contact networks, and also explore the effect of system size and contact number.

For all systems, we have found that the ensemble yields force distributions that decay much faster than exponentially. The dimensionality of the system is crucial, while other factors hardly affect the asymptotics: $P(f)$ decays as $\exp \left(-c f^{\alpha}\right)$, with $\alpha=2.0 \pm 0.1$ in two dimensions, while in three dimensions $\alpha=1.7 \pm 0.1$ [22].

Our results thus underline the importance of the additional constraints that forces have to balance on each grain: these are ignored in the "Boltzmann"-type arguments, but completely alter the properties of $P(f)$.

The ensemble approach to force networks is inspired by the proposal of Edwards to assign an equal probability to all "blocked" states, i.e., states that are at mechanical equilibrium [23]. By limiting the Edwards ensemble to a single packing of fixed contact geometry [24], where the contact forces are the remaining degrees of freedom and all allowed force configurations are sampled with equal weight, one obtains the force network ensemble. Here we restrict ourselves to spherical particles with frictionless contacts, so that every contact force $f_{i}$ corresponds to one scalar degree of freedom. Furthermore, we require all $f_{i} \geqslant 0$ due to the repulsive nature of the contacts. As the equations of mechanical equilibrium are linear in the contact forces, one can cast the solutions $\vec{f}$ $=\left(f_{1}, f_{2}, \ldots\right)$ in the form $\vec{f}=\vec{f}_{0}+\Sigma_{k} c_{k} \vec{v}_{k}$. The solution space is spanned by the vectors $\vec{v}_{k}$ and $\vec{f}_{0}$, and can be sampled through the coefficients $c_{k}$-for details we refer to Refs. $[15,16,19]$. For a hexagonal packing (two dimensional), these vectors are easily constructed using so-called wheel moves [19], but for other packings we have obtained $\vec{v}_{k}$ and 

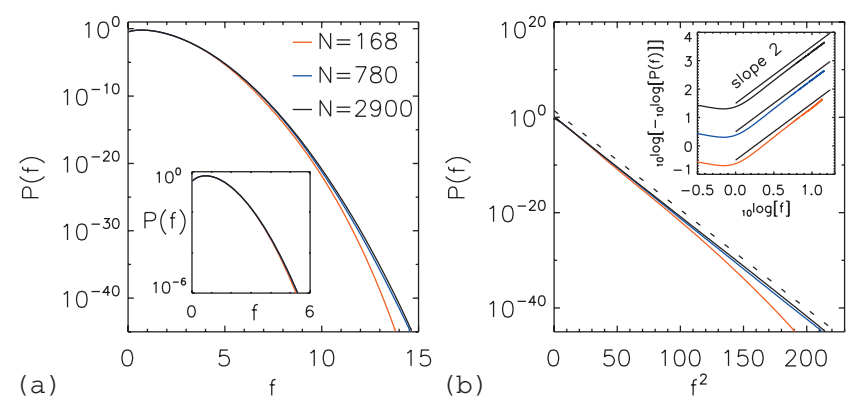

FIG. 1. (Color) Force probabilities in two-dimensional hexagonal packings of $N$ particles with periodic boundary conditions. (a) $P(f)$ decays much faster than exponentially, and rapidly converges to its asymptotic form with $N$-larger $N$ correspond to wider distributions. The inset illustrates that system size effects are hardly visible for $P(f)$ down to $10^{-6}$. (b) $\log _{10} P(f)$ vs $f^{2}$ becomes a perfectly straight line for large systems, indicating that the tail of $P(f)$ is well described by a Gaussian decay $\sim \exp \left(-c f^{2}\right)$ (dashed line). The inset shows that, on a triple-logarithmic plot, the asymptotic decay attains a slope close to 2, confirming the Gaussian tail (see text). Curves are offset for clarity, and lines are guides to the eye.

$\vec{f}_{0}$ from a simulated annealing procedure [15]. Ensemble averages using a uniform measure in this force space then become

$$
\langle q\rangle=\Omega^{-1} \int_{\mathcal{C}} d \vec{c} q, \quad \Omega \equiv \int_{\mathcal{C}} d \vec{c},
$$

where the integral runs over the coefficients $c_{k}$ limited to the convex subspace $\mathcal{C}$ for which all $f_{i} \geqslant 0$ [18].

To obtain accurate statistics for large forces, we perform umbrella sampling. The idea is to bias the numerical sampling toward solutions with large forces, using a Monte Carlo technique with a modified measure $d \vec{c} \rho(\vec{c}) / \Omega$, and then correct for this bias when performing the averages, $\langle q\rangle$ $=\langle q / \rho\rangle_{\text {umbrella }}$, since

$$
\langle q\rangle=\Omega^{-1} \int_{\mathcal{C}} d \vec{c} \rho(\vec{c})\left(\frac{q}{\rho(\vec{c})}\right) .
$$

Defining $f_{\text {max }}$ as the largest force for a given $\vec{c}$, we have used a measure $\rho(\vec{c}) \propto e^{W\left(f_{\max }\right)}$, where $W$ is chosen such that the
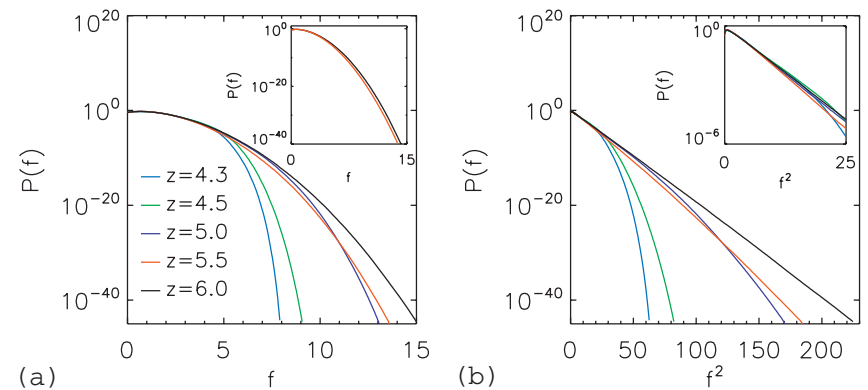

probability of $f_{\max }$ in the modified ensemble is approximately flat in the range $1<f_{\max }<15$ (the precise form of $W$ is irrelevant). We have verified that this procedure exactly reproduces $P(f)$ in the range accessible by the conventional unbiased sampling [19]. However, forces of the order of 15 are now sampled only $10^{4}$ times less frequently than forces around 1, even though their relative probability is about $10^{-45}$, leading to the spectacular improvement in numerical accuracy [25].

A well-studied geometry for which the force network ensemble yields nontrivial results is that when all particles are of equal size and form a hexagonal lattice $[15,16,19]$. The umbrella sampling allows us to access the statistics beyond $f=5$. Figure 1 (a) shows that $P(f)$ decays much faster than exponentially, and that effects of the finite size of the system are weak. Figure 1(b) illustrates that, for increasingly large systems, $P(f)$ rapidly converges to an asymptotic form which is characterized by a purely Gaussian decay. This can also be seen in the inset of Fig. 1(b), where we exploit the fact that we have access to $P(f)$ over more than 40 decades: Assuming that, for large $f, P(f) \sim \exp \left(-c f^{\alpha}\right)$, one can infer the exponent $\alpha$ from the asymptotic slope of a triple-logarithmic plot in which $\log _{10}\left(-\log _{10} P\right)$ is plotted as function of $\log _{10} f$ [4]. The inset of Fig. 1(b) shows that $\alpha=2.0 \pm 0.1$, confirming that the tail of $P(f)$ is well described by a Gaussian decay [26].

To investigate the effect of packing disorder and coordination number $z$, we have created packings from molecular dynamics simulations of soft particles in periodic boundary conditions (see $[15,17]$ ). The coordination number $z$ is controlled by the pressure in the simulations. Once a packing is obtained, all particle positions are kept fixed, and we subsequently explore the ensemble of force networks for these packings. At this point the interparticle potential is no longer used, so that grain rigidity is not a parameter in the ensemble.

For all 2D disordered packings, $P(f)$ decays much faster than exponentially, as shown in Fig. 2. Comparing the ordered hexagonal packings to a disordered system with equal coordination number, $z=6$, we find nearly indistinguishable $P(f)$ [inset Fig. 2(a)]. This suggests that the packing (dis)order and preparation history are not important for $P(f)$ in the ensemble. However, the contact number influences the
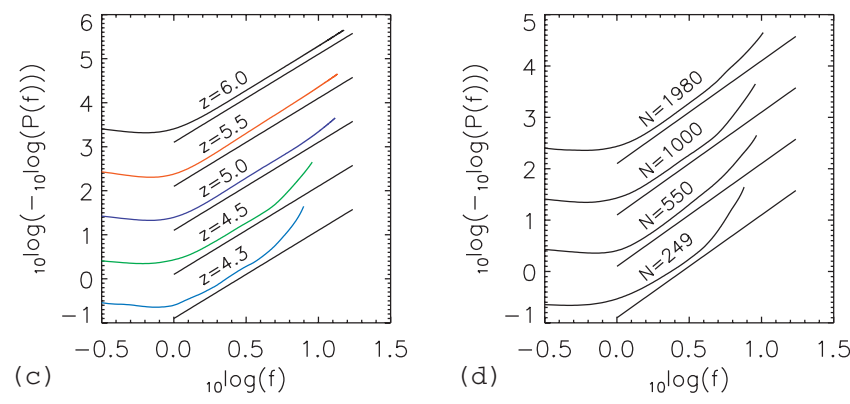

FIG. 2. (Color) Force distribution for two-dimensional systems. (a) For increasing values of the contact number $z, P(f)$ grows in width (disordered packings, $N=1000$ ). The inset compares $P(f)$ for a disordered packing with $z=6$ and $N=1000$ and the hexagonal packing for $N=2900$. (b) The same data as in (a), now plotted as $\log _{10} P(f)$ vs $f^{2}$, tends to a straight curve for large $z$. The inset shows that on a smaller range, all curves look Gaussian. (c) Same data as in (a) and (b), now on a triple-logarithmic plot. The range in $f$ over which $P(f)$ looks Gaussian grows with contact number $z$. (d) For fixed small $z=4.5, P(f)$ appears to approach a Gaussian tail for large $N$. 


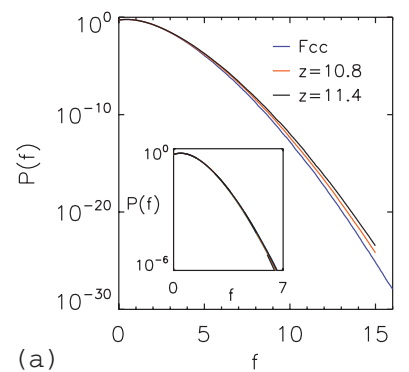

(a)
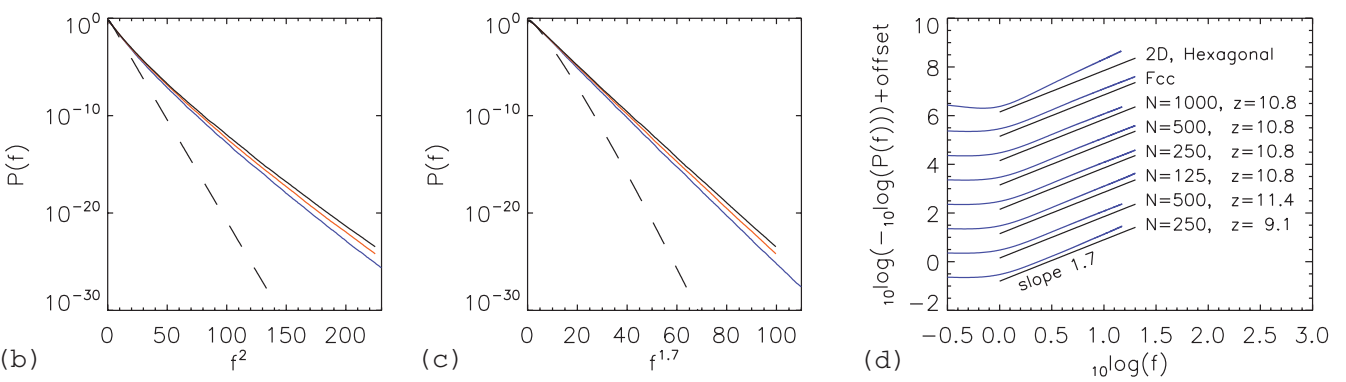

FIG. 3. (Color) Force distribution for three-dimensional systems. (a) $P(f)$ for two disordered and a regular fcc packing of $N=500$ particles - the fcc packing has the smallest width, while for the disordered packs the width grows with contact number. (b) The same, now plotted as function of $f^{2}$. The dashed line corresponds to a hexagonal packing in 2D, which has a Gaussian tail-the tail of $P(f)$ for 3D systems is significantly less steep. (c) Same data, now plotted as function of $f^{1.7}$ - the tails for the $P(f)$ of 3D packings are now straight. (d) The change from 2 to 1.7 is also clearly visible in the triple-logarithmic plot. For a range of system sizes and contact numbers, we robustly find that $P(f) \sim \exp \left(-c f^{\alpha}\right)$ with an exponent $\alpha \approx 1.7$ for 3D systems-for comparison, we also show the Gaussian distribution for the 2D hexagonal packing. Note that, for small systems and small contact number $(N=250, z=9.1)$, finite-size deviations, similar to those observed in two dimensions, can be seen.

asymptotic decay: a lower $z$ leads to a faster decay [Figs. 2(a) and 2(b)], although in the restricted range $f<5$, the force distribution appears very close to Gaussian for all $z$ [inset Fig. 2(b)]. For the lowest $z$ in particular, this tendency is cut off at large $f$, which can be clearly seen in the triplelogarithmic plot [Fig. 2(c)], where all curves tend toward a well-defined slope $\alpha=2.0$ for intermediate $f$, but cross over to a much faster decay for large $f$. We suggest that this is a finite-size effect, which is most severe when $z$ approaches the isostatic point $(z=4)$, where there are fewer and fewer degrees of freedom available [17,27]. Indeed, data for $z$ $=4.5$ and increasing system sizes suggest that the "kink" in the triple-logarithmic plots becomes less severe for large systems [Fig. 2(d)] —our data are not conclusive as to whether this kink will disappear for $N \rightarrow \infty$.

In conclusion, for two-dimensional, frictionless systems, the ensemble approach yields force distributions $P(f)$ that decay at least as fast as a Gaussian.

We now turn to three-dimensional systems, which again have been generated using molecular dynamics. Similar to what happens in two dimensions, Fig. 3(a) shows that $P(f)$ decays faster than exponentially, and disordered and regular (fcc) packings have very similar force distributions. However, the decay is now slower than Gaussian and much more accurately described by $P(f) \sim \exp \left(-c f^{\alpha}\right)$ with an exponent $\alpha=1.7 \pm 0.1$ [see Figs. 3(b)-3(d)]. This exponent has been determined from the triple-logarithmic plots of Fig. 3(d) for a range of contact numbers and system sizes, and in all cases the slope is close to $\alpha=1.7$ over a decade.

For comparison we have, in Figs. 3(b)-3(d), also included the result for the hexagonal pack, which is seen to decrease significantly more rapidly than the $P(f)$ 's of the threedimensional systems. Surprisingly, we thus find that the dimensionality of the packing determines the nature of the tail of $P(f)$.

From experiments on (two-dimensional) sheared packs of photoelastic grains, it was found that the distribution broadened significantly, and developed an exponential-like regime in a range up to $f=4\langle f\rangle[7]$. The ensemble indeed reproduces this qualitative feature for packs under shear. As can be seen in Fig. 4, however, there does not seem to be a simple asymptotic decay. This is because the force anisotropy induced by the shear stress yields a variation in $\langle f\rangle$ depending on the orientation of the contact $[17,19]$. The total $P(f)$ becomes a superposition over all orientations, of mixed force statistics, and hence lacks a single characteristic feature.

We have shown for the force network ensemble that the tail of $P(f)$ decays faster than exponentially, in agreement with recent experiments $[7,9]$, but inconsistent with others [2-4]. Some of our results required extremely accurate statistics, beyond the regime accessible by experiments or conventional simulations. In particular, it would be difficult to distinguish an exponent $\alpha=1.7$ from 2.0. Nevertheless the discrepancy between the exponential data sets [2-4] and the faster-than-exponential data sets $[7,9]$ cannot be explained away by finite error bars, but is convincing and worthwhile of further investigations.

The experimental and numerical data for $P(f)$ have been obtained from a wide variety of systems and models, and parameters such as dimensionality, friction, hardness of grains [28], and bulk vs boundary measurements may ulti-
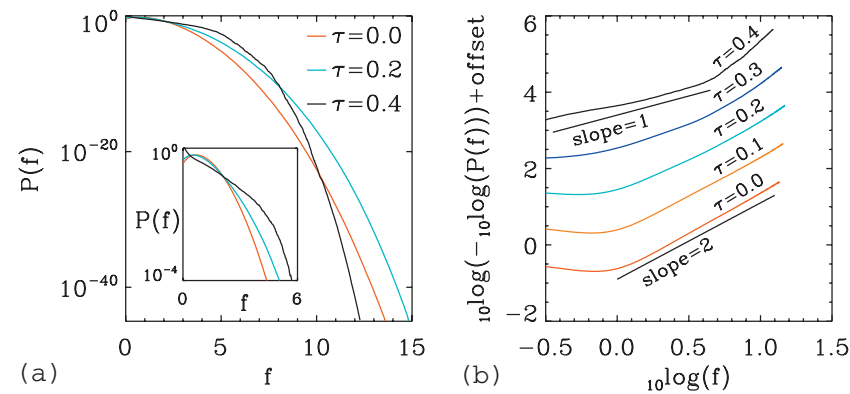

FIG. 4. (Color) Two-dimensional disordered system with $z$ $=5.5$ experiencing a shear stress $\tau \equiv \sigma_{x y} / \sigma_{x x}$ [17]. (a) While, for large $\tau$, the tail of $P(f)$ viewed over a limited range broadens and may appear exponential (inset), the asymptotic decay of $P(f)$ for $f>10$ in fact increases with $\tau$ (main panel). (b) The same point is illustrated in the triple-logarithmic plots, which also show data for $\tau=0.1$ and 0.3 . 
mately all play a role in determining the asymptotics of $P(f)$. The ensemble can also be extended to include torque balance and to explore boundary measurements. Based on earlier work on boundary effects [29], however, we speculate that these extensions would not alter the tail of $P(f)$ significantly.

A crucial untested assumption in the ensemble is the flat measure, i.e., the sampling of all allowed configurations with equal weight. As argued in Ref. [4], different experimental procedures and parameters can lead to a different $P(f)$, so that the effective sampling of force networks may not be universal.

W.G.E acknowledges financial support from the physics foundation FOM, M.v.H. and T.J.H.V. from NWO/VIDI, and J.H.S from a Marie Curie European Action FP6 (Grant No. MEIF-CT-2006-025104).
[1] H. M. Jaeger, S. R. Nagel, and R. P. Behringer, Rev. Mod. Phys. 68, 1259 (1996).

[2] C. Liu et al., Science 269, 513 (1995); D. M. Mueth, H. M. Jaeger, and S. R. Nagel, Phys. Rev. E 57, 3164 (1998); D. L. Blair, N. W. Mueggenburg, A. H. Marshall, H. M. Jaeger, and S. R. Nagel, ibid. 63, 041304 (2001); J. M. Erikson, N. W. Mueggenburg, H. M. Jaeger, and S. R. Nagel, ibid. 66, 040301 (2002).

[3] G. Løvoll, K. J. Måløy, and E. G. Flekkøy, Phys. Rev. E 60, 5872 (1999).

[4] E. I. Corwin, H. M. Jaeger, and S. R. Nagel, Nature (London) 435, 1075 (2005).

[5] H. A. Makse, D. L. Johnson, and L. M. Schwartz, Phys. Rev. Lett. 84, 4160 (2000).

[6] F. Radjai, M. Jean, J. J. Moreau, and S. Roux, Phys. Rev. Lett. 77, 274 (1996); C. S. O'Hern, S. A. Langer, A. J. Liu, and S. R. Nagel, ibid. 86, 111 (2001); A. V. Tkachenko and T. A. Witten, Phys. Rev. E 62, 2510 (2000); L. E. Silbert, G. S. Grest, and J. W. Landry, ibid. 66, 061303 (2002).

[7] T. S. Majmudar and R. P. Behringer, Nature (London) 435, 1079 (2005).

[8] J. Brujic, S. F. Edwards, I. Hopkinson, and H. A. Makse, Physica A 327, 201 (2003).

[9] J. Zhou, S. Long, Q. Wang, and A. D. Dinsmore, Science 312, 1631 (2006).

[10] S. N. Coppersmith, C.-h. Liu, S. Majumdar, O. Narayan, and T. A. Witten, Phys. Rev. E 53, 4673 (1996).

[11] P. Claudin, J.-P. Bouchaud, M. E. Cates, and J. P. Wittmer, Phys. Rev. E 57, 4441 (1998).

[12] N. P. Kruyt and L. Rothenburg, Int. J. Solids Struct. 39, 571 (2002); K. Bagi, Granular Matter 5, 45 (2003).

[13] J. Rottler and M. O. Robbins, Phys. Rev. Lett. 89, 195501 (2002).

[14] P. T. Metzger, Phys. Rev. E 70, 051303 (2004).

[15] J. H. Snoeijer, T. J. H. Vlugt, M. van Hecke, and W. van Saarloos, Phys. Rev. Lett. 92, 054302 (2004).

[16] J. H. Snoeijer, T. J. H. Vlugt, W. G. Ellenbroek, M. van Hecke, and J. M. J. van Leeuwen, Phys. Rev. E 70, 061306 (2004).

[17] J. H. Snoeijer, W. G. Ellenbroek, T. J. H. Vlugt, and M. van
Hecke, Phys. Rev. Lett. 96, 098001 (2006).

[18] T. Unger, J. Kertész, and D. E. Wolf, Phys. Rev. Lett. 94, 178001 (2005).

[19] B. P. Tighe, J. E. S. Socolar, D. G. Schaeffer, W. G. Mitchener, and M. L. Huber, Phys. Rev. E 72, 031306 (2005).

[20] S. Ostojic and D. Panja, Phys. Rev. Lett. 97, 208001 (2006); S. Ostojic, M. van Hecke, and B. Nienhuis (unpublished).

[21] D. Frenkel and B. Smit, Understanding Molecular Simulation: From Algorithms to Applications (Academic, San Diego, 2002).

[22] Similar exponents emerge for the potential energy of Hertzian contacts [4], which scale as $f^{2}$ and $f^{5 / 3}$, respectively. As the ensemble considers rigid particles without any contact law, this appears to be a coincidence.

[23] S. F. Edwards and R. B. S. Oakeshott, Physica A 157, 1080 (1989).

[24] J. P. Bouchaud, in Slow Relaxations and Nonequilibrium Dynamics in Condensed Matter, edited by J. L. Barrat, M. Feigelman, J. Kurchan, and J. Dalibard, Proceedings of the Les Houches Summer School of Theoretical Physics, LXXVII (EDP Sciences, Ulis, 2003).

[25] A. R. T. van Eerd and T. J. H. Vlugt (unpublished).

[26] Intriguingly, the force distribution is surprisingly well approximated at small $f$ by $P(f) \simeq 1 / 3+3 f / 4$, which hints that a simple analytic expression may exist for the hexagonal packing. A decent fit over the whole range of $f$ is given by $P(f)$ $=(a+b f) e^{-c\left(f-f_{0}\right)^{2}}$, where $a, b$ are determined by the observed small- $f$ behavior, and $f_{0}, c$ are determined by $\langle f\rangle=1$ and $\int P(f) d f=1$, but deviations from the numerical $P(f)$ can be observed in the tail.

[27] C. F. Moukarzel, Phys. Rev. Lett. 81, 1634 (1998); A. V. Tkachenko and T. A. Witten, Phys. Rev. E 60, 687 (1999).

[28] Note that the glass beads used in the boundary measurements are much harder than the particles used in the bulk measurements of [7-9], and that realistic particle rigidity is difficult to achieve in molecular dynamics.

[29] J. H. Snoeijer, M. van Hecke, E. Somfai, and W. van Saarloos, Phys. Rev. E 70, 011301 (2004). 